\title{
\begin{tabular}{l|l} 
POLITIQUES \& & Politiques et management public
\end{tabular}

\section{Un système de pilotage de la performance publique à dominante managériale : analyse de l'expérience de Dubaï}

A public performance piloting system with manager dominant feature: analysis of Dubai experiment

\section{Bouchra Fninou and François Meyssonnier}

\section{OpenEdition}

\section{Journals}

\section{Electronic version}

URL: http://journals.openedition.org/pmp/6686

ISSN: 2119-4831

Publisher

Institut de Management Public (IDPM)

\section{Printed version}

Date of publication: 15 June 2013

Number of pages: 241-259

ISBN: 978-2-7430-1538-1

ISSN: 0758-1726

\section{Electronic reference}

Bouchra Fninou et François Meyssonnier, « Un système de pilotage de la performance publique à dominante managériale : analyse de l'expérience de Dubaï », Politiques et management public [En ligne], Vol 30/2 | 2013, mis en ligne le 28 septembre 2015, consulté le 30 avril 2019. URL : http:// journals.openedition.org/pmp/6686 


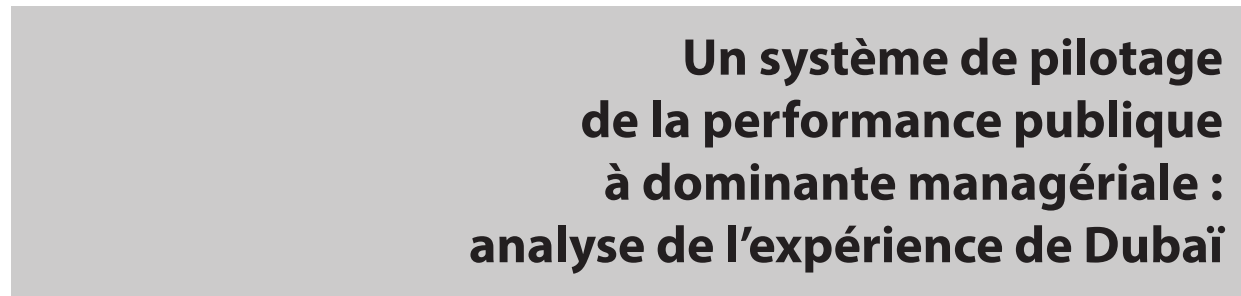

Bouchra Fninou ${ }^{a}$ et François Meyssonnier ${ }^{b^{*}}$

a Budget manager, Gouvernement de Dubaï, Émirats Arabes Unis

Doctorante en Sciences de Gestion à l'Université de Nantes

${ }^{b}$ Professeur de Sciences de Gestion, LEMNA - IAE-Université de Nantes

Chemin de la Censive du Tertre - 44000 Nantes

\section{Résumé}

Le mode de gestion de l'administration publique de Dubaï est très spécifique. Après avoir présenté les caractéristiques de la cité-État et les facteurs de contingence s'exerçant sur son administration, le système de pilotage de la performance publique de Dubaï, fondé sur les principes du New Public Management est décrit et analysé. L'article montre la cohérence, les apports et les limites de cette démarche emblématique d'une modernisation de l'administration publique fondée sur la culture du résultat.

๑) 2013 IDMP/Lavoisier SAS. Tous droits réservés

Mots clés : new public management, système de pilotage de la performance publique, facteurs de contingence, administration territoriale, Dubaï.

\section{Abstract}

À public performance piloting system with manager dominant feature : analysis of Dubai experiment. The style of management of Dubai government is very particular. After presenting the characteristics of the city-state and contingency factors acting on its administration, the control system of the public performance of Dubai, based on the principles of New Public Management is described and analyzed. The article shows consistency, contributions and limits of this emblematic approach of public administration modernization based on the results-oriented culture.

(c) 2013 IDMP/Lavoisier SAS. Tous droits réservés

Keywords: new public management, public performance management system, contingency factors, territorial administration, Dubai.

*Auteur correspondant : francois.meyssonnier@univ-nantes.fr doi :10.3166/pmp.30.241-259 @ 2013 IDMP/Lavoisier SAS. Tous droits réservés 


\section{Introduction}

Certains auteurs estiment que la gestion publique a des spécificités fortes par rapport à la gestion des entreprises privées. Ils justifient ainsi une approche qui privilégie la dimension macro des politiques publiques globales, les enjeux de gouvernance et les aspects sociopolitiques de la gestion publique. La tradition académique classique française, telle qu'elle s'exprime largement dans la revue « Politiques et Management Public », en est une descendante naturelle. La conception « française » de l'évaluation des politiques publiques atteste par exemple des fortes réticences des responsables publics face à la « culture du résultat » et à l' "accountability » et se caractérise par l'accent mis sur les aspects de « légitimité » et de "complexité ». C'est le point de vue dominant actuellement dans la communauté francophone de chercheurs en management public.

D'autres auteurs estiment que la gestion des organisations publiques a des caractéristiques propres, contextuelles mais relève du même cadre d'analyse que les organisations privées en matière de science de gestion. Certes la gestion d'une mairie n'est pas exactement de même nature que la gestion d'un hypermarché mais la gestion d'un hypermarché est de son côté elle aussi assez différente de celle d'une usine sidérurgique ou d'une start-up de la nouvelle économie numérique. Les contextes diffèrent. Les facteurs de contingence varient. Les principes managériaux universels demeurent et s'appliquent aussi aux entités publiques pensent les tenants du New Public Management (Hood, 1991). Pour ces auteurs, il faut s'intéresser autant à l'usager qu'au citoyen, autant à l'efficience et à l'efficacité qu'à la participation citoyenne. Cette approche privilégie la « value for money », l'équilibre des finances publiques, la productivité des services publics au niveau micro, la satisfaction des usagers prestation par prestation. La spécificité du management public est alors évidente dans la conception des politiques publiques mais pas forcément fondamentale dans leur mise en œuvre concrète (Meyssonnier, 1997). Cette conception est actuellement minoritaire en France dans les associations et revues spécifiques.

La présentation d'une étude sur la conception et la mise en œuvre d'un Système de Pilotage de la Performance Publique (SPPP dorénavant) d'une administration très particulière permettra d'apporter une contribution utile à ces échanges. La comparaison des expériences en matière de gestion des collectivités territoriales est une étape indispensable dans la recherche d'une plus grande efficacité de la dépense publique afin de voir les réussites et les échecs, les leçons généralisables et les aspects contingents. Mais elle est difficile. Parfois on apprend plus de l'étude approfondie d'un cas atypique ou d'une situation spéciale que de la répétition d'études similaires fondées sur toujours la même population d'organisations. C'est pourquoi, au lieu d'étudier classiquement les pratiques de gestion des collectivités territoriales dans un grand pays occidental, nous analyserons les modes de gestion d'une cité-État emblématique du monde contemporain : Dubaï. Il s'agit d'une recherche exploratoire et descriptive sur la nature du SPPP de Dubaï. Ce SPPP est très fortement inspiré par l'approche du New Public Management. L'étude de ce mode de gestion particulier semble susceptible d'éclairer les transformations contemporaines du management public en faisant sa place à une collectivité territoriale caractéristique de la modernité dans la mondialisation. En effet, Dubaï joue un rôle financier et commercial spécifique et éminent au Moyen-Orient comparable à celui de Luxembourg en Europe ou de Singapour en Asie du Sud-Est. 
Après avoir présenté la problématique et le terrain de la recherche (1), de la cité-État l'impact de certaines spécificités du contexte de Dubaï sur les modes de gestion de l'administration de la cité-État sera décrit (2). Ensuite, la nature du SPPP de Dubaï, marqué par la tradition anglo-saxonne du New Public Management, sera soulignée (3). Ceci permettra d'analyser ce mode de gestion mis en œuvre dans un contexte particulier avec des résultats indubitables et certaines limites.

\section{L'étude du système de pilotage de l'administration de Dubaï}

S'il est courant dans le cadre des études de cas qualitatives et descriptives de s'intéresser aux difficultés rencontrées en matière de changement et de modernisation dans les vieilles administrations publiques des grands États nationaux d'Europe, dont la France est un exemple archétypal, il est assez rare de décrire la façon dont une administration publique, se construisant quasiment à partir de rien et accompagnant l'émergence d'une cité-État insérée dans la globalisation, structure son SPPP. C'est cette démarche qui va maintenant être présentée.

\subsection{Une recherche s'inscrivant dans le cadre des sciences de gestion}

Zampiccoli (2011) effectue une typologie des conceptions du management public selon deux axes : la prise en compte plus ou moins grande des particularités des organisations publiques et la dynamique plus ou moins forte de transformation du système. Cela l'amène à distinguer quatre positionnements dans la conception du management public :

- le « management face à l'opinion » qui concerne aussi bien le privé que le public et qui fait de la responsabilité sociétale, de la communication et de la légitimation vis-à-vis des tiers des enjeux centraux (Laufer et Burlaud, 1980);

- le «management du pouvoir politique » qui s'intéresse aux interactions entre politiques et administratifs dans le pilotage des organisations publiques et aux processus de prise de décision et qui relève du courant de la complexité en sciences de gestion; - le «management de la puissance publique » qui insiste sur la double fonction de production des organisations publiques et sur l'impact des politiques publiques (Gibert, 1986);

- le «management des organisations publiques » ou New Public Management qui s'intéresse à l'efficacité et l'efficience des processus serviciels aux usagers (Meyssonnier et Tahar, 2011).

Les trois premières conceptions s'appuient (essentiellement dans les deux premiers cas, de façon plus modérée pour l'approche «management de la puissance publique ») sur la sociologie, les sciences politiques, les théories de la communication. In fine, ces visions sont assez proches dans le positionnement sur le terrain et dans les préconisations. La dernière conception est fondée principalement sur les sciences économiques. En matière de modernisation du secteur public, elle développe un ensemble de préconisations très largement alternatives aux précédentes. C'est ce dernier cadre de référence qui a été retenu pour cette étude consacrée à une administration gérée comme une entreprise et qui se transforme en permanence. 


\subsection{La question de la nature des systèmes de pilotage de la performance publique}

La recherche vise à décrire les différents traits du SPPP de la cité-État de Dubaï et à analyser les différents aspects de sa conception et de son implémentation. Dans ce cadre, l'instrumentation de gestion déployée et les systèmes d'information et d'animation activés dans l'administration de Dubaï seront analysés. La question de recherche est la suivante : «Quelles sont les caractéristiques et les spécificités du SPPP de la cité-État de Dubaï? ». Il s'agit de décrire et d'étudier les éléments qui composent ce SPPP et de voir comment concrètement les choses fonctionnent. Le matériel de base va être l'ensemble des documents officiels et archives disponibles et des entretiens avec des cadres dirigeants de l'administration de Dubaï. Les déclarations seront recoupées et confrontées entre elles et à toute une série d'éléments et documents à notre disposition, ce qui permettra d'en avoir une vision critique. À cette étape de la recherche, la question de l'appropriation de l'instrumentation de gestion par les managers et personnels de terrain ne sera qu'effleurée. De même, les problèmes de gouvernance, de relations entre l'autorité politique et le staff administratif de direction seront laissés de côté. L'analyse s'intéresse essentiellement à la cohérence d'ensemble du mode de pilotage, à la façon dont ses composantes sont articulées et à sa logique intrinsèque. L'apport attendu est la compréhension des aspects universels et des aspects contingents des SPPP fondés sur le New Public Management.

\section{Le micro-État de Dubaï}

La population de Dubaï est d'environ deux millions de résidents (3/4 d'hommes, $1 / 4$ de femmes) dont moins de $10 \%$ de nationaux. Le PIB par habitant de Dubaï est comparable à ceux de Singapour ou de Hong-Kong. La croissance de ce ratio a été de $6 \%$ par an pour la période 2000-2005 combinant une croissance du PIB (hors pétrole) de $15 \%$ et une progression de la population de $7 \%$ par an en moyenne. Les grands domaines d'activité du hub que constitue Dubaï sont : les voyages et le tourisme; les services financiers; les services professionnels; le transport et la logistique; le commerce et les échanges ; l'immobilier et quelques industries. La cité-État a opté pour une diversification économique et ne dépend plus des revenus du pétrole qui représentent moins de $15 \%$ du PIB.

Plusieurs zones franches ont été constituées qui attirent les entreprises multinationales par les avantages qu'elles offrent : facilité d'installation, exonération d'impôts, droits de douane très faibles, rapatriement possible de $100 \%$ des bénéfices ; facilités de recrutement, environnement de travail agréable, etc. Les plus connues de ces zones franches sont : «Media City » où sont installées des antennes régionales de Reuters, CNN, MBC...; «Internet City » où sont installées des entreprises de haute technologie comme IBM, Dell, Sony, Hewlett Packard...; «Dubä̈ International Academic City » où cohabitent 37 campus internationaux d'universités relevant de 11 nationalités (Australie, Canada, États-Unis, France, Grande-Bretagne, Inde, Liban, Pakistan, Russie, etc.) Cette dernière zone franche vise à développer les talents de la région et à faire de Dubaï une économie fondée sur la connaissance (Knowledge-Based Economy). En matière de main-d'œuvre, Dubaï recourt à un système de sponsorship 
appelé « kafala : les étrangers sont placés sous la responsabilité et la protection d'un «kafil». Les étrangers soumis à la «kafala» peuvent à tout moment être renvoyés et cela entraîne souvent un sentiment d'infériorité et de mise sous tutelle.

Depuis la fondation des Émirats Arabes Unis en 1971, Dubaï a structuré progressivement son appareil administratif propre qui est passé de 45000 fonctionnaires en 2005 à un peu plus de 80000 en 2011. À la tête de chacune des administrations de Dubaï on trouve des hauts fonctionnaires émiratis nommés par le Cheikh Mohamed Ben Rachid Al Maktoum, tous issus des grandes familles, riches et connues, de l'émirat (Al Maktoum, Al Marri, Al Tayer, Al Qamzi, etc.) Le conseil exécutif qui assiste le Cheikh a développé un plan de développement stratégique à horizon 2015 avec cinq volets (Gouvernement de Dubaï, 2011) : le développement économique (14\% du budget en 2010) ; le développement social (22\% du budget); les infrastructures et équipements (45\% du budget); la sécurité et la justice (19\% du budget); la recherche de l'excellence dans l'administration. Les objectifs assignés à ce dernier volet sont : la transparence; la performance financière; une culture du résultat; le service à l'usager; le développement des ressources humaines; le respect du cadre fédéral des Émirats.

Le recours aux mécanismes de marché pour assurer les prestations nécessaires à la population est généralisé. Par exemple, les services sociaux d'éducation et de santé sont fournis pour une petite partie par l'administration publique et pour l'essentiel par des opérateurs privés. Ainsi seulement $12 \%$ de l'ensemble des élèves scolarisés à Dubaï le sont dans les établissements publics (en même seulement $43 \%$ des enfants émiratis).

Dans le champ social, le rôle de l'administration publique est plus le contrôle et la certification que de mise en œuvre directe. Les prestations dans le secteur public sont gratuites pour les émiratis et au prix du marché pour les expatriés. Fondamentalement, le gouvernement de Dubaï est plus intéressé par les activités de régulation et de certification de la qualité des services aux citoyens que par la mise en œuvre directe des prestations qui sont la plupart du temps réalisées par des entreprises privées dans le domaine de l'éducation et de la santé.

\subsection{Une observation participante approfondie}

Les très petits États (Glassner, 2004) ont des caractéristiques de développement spécifiques (Armstrong et al., 1998; Heard-Bey, 1999; Crowards, 2002) et rencontrent des difficultés contingentes (Benedict, 1967 ; Katzenstein, 2003; Drieskens, 2007). Leur administration publique doit faire face à des enjeux et problématiques de gestion particuliers (Farrugia, 1993 ; Thorhallsson, 2000 ; Sevic, 2001 ; Randma-Liiv, 2002 a et b ; Curmi, 2009; Sarapu, 2010). Notre étude s'appuie sur différentes études portant soit sur la cité-État de Dubaï (Marchal, 2001 ; Dumortier et Lavergne, 2002; Lavergne 2002 et 2009; Davidson, 2008; El Shimy, 2008; Oxford Business Group, 2008; Vine, 2010 ; Dubaï Statistics Center, 2010), soit sur les modes de management de son administration publique (Makharita, 2005; Lootah et Okan, 2006; Elashmawy, 2007 ; Department of Finance, 2009 a et b ; El-Kahlout, 2010 ; Biygautane et al., 2010 et 2011 ; Government of Dubaï, 2011). 
Les observations sont fondées sur une recherche-observation sur la durée, puis sur des entretiens. L'étude a été menée à partir d'une collecte systématique de tous les documents disponibles sur place (depuis le lancement de la réforme budgétaire de 2007) et des entretiens avec dix cadres dirigeants de six services administratifs représentatifs de l'administration dubaïote : éducation (KHDA - Knowledge and Human Development Authority), transport (DA - Dubaï Airports), développement économique (DED - Dubaï Economic Development), sécurité (DP - Dubaï Police), état-civil (DNRD - Department of Naturalisation and Residency Dubai), finances (DOF - Department of Finance). Les entretiens ont duré deux heures en moyenne et se sont déroulés en arabe (six entretiens), en anglais (trois entretiens) ou en français (un entretien) de février à avril 2012. Les cadres font partie de l'équipe de direction de l'administration de Dubaï et ont des responsabilités de directeur général du service, de directeur de l'évaluation de la performance (contrôle de gestion) ou de directeur financier. Ils sont Émiratis (six d'entre eux) ou expatriés (quatre dont un italien, deux Égyptiens et un Jordanien). Le détail est donné en annexe.

Les chercheurs sont un cadre supérieur arabophone de l'administration de Dubaï en poste depuis plusieurs années (ce qui permet la compréhension de l'intérieur des caractéristiques et du contexte de l'organisation) et un universitaire européen assurant l'encadrement de l'étude dans le cadre d'une thèse (ce qui garanti l'encadrement méthodologique et le recul nécessaire à une telle recherche de terrain).

Après cette présentation du design de la recherche, le cadre à l'intérieur duquel le SPPP de Dubaï se déploie va être spécifié.

\section{Contexte et caractéristiques de la gestion publique de Dubaï}

L'administration de la cité-État de Dubaï est marquée par une petite taille, un multiculturalisme aussi bien dans la population que dans le personnel administratif, le « clientcentrisme » et une forte inspiration des méthodes de gestion des entreprises privées.

\subsection{Une organisation administrative de petite taille}

La petite taille du pays et de son administration a des avantages :

«Les petits États atteignent leurs objectifs rapidement, leurs problèmes sont peu nombreux et partant ils peuvent trouver des solutions assez vite. Quand le pays est vaste et ancien, les traditions sont fortes, le changement requiert beaucoup d'efforts et les fraudes sont nombreuses. Quand le pays est plus petit, le périmètre de travail est plus réduit, on peut y faire plus de contrôle, on peut déterminer ses objectifs plus facilement, on peut définir les activités demandées d'une manière plus claire, la détermination des coûts est plus facile et les fraudes sont réduites. Pour rendre quelqu'un accountable sur son programme, on lui demande de fournir ses indicateurs de performance. Si le programme est bien délimité et bien spécifique et que son ampleur est faible, les chances de réussite sont importantes. » (DOF-M.A).

Ceci permet un fonctionnement proche du terrain :

"Une chose que nous avons décidée au sein de KHDAc'est ce que l'on appelle "Nahjouna » ou bien la manière de faire les choses. On fait peu de chose mais on les fait bien.» (KHDA - Docteur D) 
Dans l'environnement turbulent de Dubaï, une amélioration permanente est indispensable : «Nous sommes dans un environnement dynamique qui est lié à la grande variation des prix du pétrole, aux tensions politiques dans la région. L'amélioration continue et l'adaptation sont essentielles. On ne peut jamais dire que ça y est, on est performant et c'est fini, car il faut toujours faire des adaptations de toutes les composantes de la performance c'està-dire une révision du programme, du but, des objectifs, des activités, des coûts et des indicateurs clés de performance (KPI).» (DOF-MA)

Le fonctionnement de l'administration de Dubaï est relativement souple :

«La stratégie évolue, elle n'est pas figée. Ils l'actualisent, ils l'améliorent. Le fait de ne plus avoir d'écoles publiques c'est un grand changement stratégique. KHDA ne l'a pas initié mais l'environnement évolue, donc on doit s'adapter. » (KHDA - M.P)

La culture du dialogue est importante et les aspects informels sont pris en compte :

"On donne beaucoup d'importance aux relations personnelles. On organise beaucoup de réunions pour discuter face à face et résoudre les problèmes. Le management est proche des employés. Les directeurs font des visites régulières à leurs employés non pas pour les contrôler mais juste pour les saluer et pour leur montrer qu'on est là pour eux » (DED - M.M).

On doit tenir compte des traditions anciennes du pays ce qui n'est toujours pas le plus productif :

"On communique beaucoup mais les informations opérationnelles sont informelles. C'est la culture du «Majlis »: on s'assoit et on discute. » (KHDA - M.P)

Des dispositifs matériels et organisationnels sont mis en place pour favoriser ces échanges : «Nous avons cassé les bureaux et supprimé les vitres pour avoir un environnement de travail ouvert qui permet beaucoup de dialogue entre les collègues. » (KHDA - Dr. K)

Il y a donc une volonté affichée de développer et partager l'information. C'est vrai par exemple dans l'administration des aéroports :

«Nous faisons des meetings chaque semaine avec nos subordonnés pour discuter de toutes les difficultés rencontrées et essayer de les résoudre. Nous encourageons nos employés à intervenir directement avec leurs collègues et à organiser des réunions. Nous faisons aussi des réunions mensuelles pour que chaque département présente ses points de vue et ses résultats. » (DA - M.R)

La petite taille de l'administration publique de Dubaï permet une flexibilité, une adaptabilité et un apprentissage continu. Le contrôle se veut plus interactif que diagnostique (Simons, 1995) avec une proximité vis-à-vis des employés, une supervision directe et un système d'information peu formel. Mais peut-être que l'importance de la dimension informelle et une trop grande bureaucratie, que déplore un des cadres dirigeants interviewés :

«Pour moi, je pense que nous avons trop de niveaux hiérarchiques et des organigrammes confus. », nuisent-ils à une certaine efficacité de gestion.

\subsection{Une grande diversité culturelle}

Les Émiratis sont une toute petite partie de la population résident à Dubaï (moins de $10 \%$ ). Les expatriés viennent du monde arabe mais aussi d'Occident et pour la plus grande part de pays d'Asie comme l'Inde, le Pakistan, le Bangladesh et les Philippines (plus des 2/3 des expatriés). La majeure partie de la population n'est donc ni arabe, ni musulmane. 
Même si les postes administratifs sont souvent attribués de préférence à des émiratis, le multiculturalisme à l'intérieur de l'administration de Dubaï est très prononcé :

«À KHDA, nous gérons et réglementons treize différents programmes d'études. Si nous n'étions que des émiratis, comment ferait-on pour bien connaître les programmes indiens, philippin, français, britannique, américain, etc.? Vous devez avoir une diversité culturelle équilibrée pour répondre aux besoins de votre activité. » (KHDA - Dr. K)

L'aspect multiculturel diffère toutefois d'une administration à une autre. La municipalité de Dubaï a été créée dans les années soixante-dix avec beaucoup de nationaux. À cette époque, Dubaï avait moins d'expatriés. Dans les administrations nouvellement créées, on trouve proportionnellement plus d'expatriés. L'administration de Dubaï emploie 50000 expatriés $(65 \%)$ et 30000 nationaux (35\%), avec plus des 3/4 d'employés hommes. La diversité culturelle de Dubaï, aussi bien dans la population que dans le personnel administratif, constitue une caractéristique marquante de la gestion publique de Dubaï :

«À Dubaï, on donne plus de liberté et de flexibilité de travail aux gens pour bénéficier de leur compétence et de leur expérience. Il y a des pays comme l'Arabie Saoudite, qui ont des expatriés mais ils ne leur donnent pas les mêmes opportunités et les mêmes libertés et partant ne bénéficient pas de la diversité culturelle.»(DOF-M.A)

Dans la diversité, il y a des aspects positifs et d'autres plus complexes à gérer (Hofstede, 1983, d'Iribarne, 1989) :

"La diversité ça apporte de la richesse à l'analyse parce que chacun perçoit les choses différemment mais il peut y avoir aussi pas mal de malentendus. Si tu as un engagement des différents groupes, c'est plutôt positif. Mais si la motivation est faible, les gens ne vont pas faire beaucoup d'effort de compréhension. » (KHDA - M.P) ou encore : «La diversité culturelle a une bonne influence sur le travail et sur la performance que ce soit en termes d'idées, d'intégration ou de travail en équipes. La différence de point de vue est positive mais parfois elle a un impact négatif sur le bon déroulement du travail car ça peut retarder le travail, influer sur la qualité, entrainer des conflits et des problèmes, ce qui rend l'environnement de travail peu motivant. » (DED - M.M)

Ainsi, des difficultés apparaissent dans le fonctionnement interne de l'administration entre les différentes nationalités :

«Parfois on est dans un meeting et les gens commencent à parler en arabe. Dubaï n'est pas un pays où l'arabe est la langue majoritaire. S'ils veulent faire comme cela, ils n'ont qu'à embaucher des arabophones! » (un cadre administratif expatrié non arabophone).

\subsection{Un fonctionnement tourné vers la satisfaction du client}

Le «client-centrisme » est au cœur du management public de Dubaï. La qualité des prestations est contrôlée et la satisfaction des usagers est mesurée :

«Nous faisons des enquêtes. À travers ces enquêtes, les employés et les clients externes font part de leurs commentaires sur les éléments à améliorer. Nous avons aussi un système de plaintes sur notre site Web. Dans notre entité, nous avons un bouton dans le bureau de chaque agent traitant avec les clients. Si le client n'est pas satisfait du service de cet agent, il peut appuyer sur ce bouton et le manager va venir pour résoudre le problème. » (DED - M.M) 
Toute une série de moyens est mise en œuvre pour cela, par exemple dans les aéroports : "Pour piloter la satisfaction des usagers, nous faisons appel à l'organisme ACI [Airports Council International] qui nous fournit un rapport détaillé sur tous les aspects du service client. Nous faisons appel aussi à d'autres institutions qui nous aident à faire des enquêtes auprès des passagers ou qui nous aident à contrôler les fluctuations journalières des passagers. Nous avons un site Web et un centre d'appel pour répondre aux demandes d'informations ou aux plaintes. Il y a aussi ce que l'on appelle le mystery shopper qui fait semblant d'être un passager normal. Il pose des questions prédéfinies à nos agents et prépare un rapport qu'il envoie à notre mangement. En se basant sur ce rapport, on essaie d'améliorer les zones de faiblesse. » (DA-M.R)

L'administration publique de Dubaï met le client au centre de ses préoccupations (Aberbach et Christensen, 2005). Un cadre remarque :

«Le gouvernement de Dubaï est devenu une entité qui dit «j'ai l'honneur de servir le citoyen » et pas "je fais une faveur au citoyen », ce qui n'est pas le cas dans plusieurs pays du Golfe analogues à Dubaï. » (DOF-M.A)

Plus que de « citoyen » (concept difficile à définir quand les nationaux représentent moins de $10 \%$ de la population) c'est d' « usager » qu'il faudrait ici parler. Cette orientation de service à l'usager permet aussi probablement de pallier en partie l'absence de démocratie à Dubaï, la faiblesse des corps intermédiaires et l'inexistence d'élus représentatifs.

\subsection{Un mimétisme de gestion par rapport aux entreprises}

À Dubaï sont installé un grand nombre d'antennes, d'agences ou de sièges régionaux d'entreprises multinationales. Par ailleurs la dynastie régnante a aussi constitué une multitude d'entreprises parapubliques intervenant dans les domaines des infrastructures portuaires, de l'immobilier, des services professionnels de haut niveau (comme la médecine ou les loisirs haut de gamme), etc. Les valeurs libérales en économie, les pratiques de gestion les plus modernes et une grande ouverture au monde sont dominantes chez les décideurs de la cité-État.

Les orientations stratégiques de l'administration sont formalisées et déclinées comme dans les entreprises. Ceci s'applique au niveau des administrations qui mettent en œuvre les choix stratégiques :

"Les indicateurs de performance sont mis dans le plan stratégique. Pour chaque objectif, il y a un indicateur, une cible et un responsable ("owner ») car il y a un suivi de cet indicateur et il y a une responsabilisation de cet «owner» ( accountability »). Chaque trimestre, les secteurs préparent un rapport incluant tous les indicateurs avec les résultats. En coordination avec le département stratégique, on présente ces résultats au comité exécutif qui juge la performance de chaque secteur ou institution. Les secteurs qui n'ont pas atteints leurs objectifs se justifient et présentent les raisons. » (DED - M.M)

Comme dans beaucoup d'entreprises privées, ceci s'incarne dans un balanced scorecard (BSC) construit pour assurer le pilotage :

«Actuellement, un BSC est mis en place par toutes les administrations de Dubaï. Nos indicateurs sont financiers et non financiers. Certains d'entre eux proviennent du gouvernement (conseil exécutif), d'autres sont discutés avec le conseil d'administration et les gestionnaires internes, puis nous sélectionnons les différents indicateurs. Les indicateurs viennent tous de 
la stratégie de KHDA. Pour chaque indicateur, nous avons une cible. Les KPI sont mesurés trimestriellement, semestriellement parfois annuellement. »(KHDA - Dr. D).

Le développement économique de Dubaï fondé sur l'implantation de multiples firmes multinationales a favorisé un mouvement de mimétisme formel en matière d'instrumentation de gestion par rapport au secteur privé et le développement de la culture du résultat.

Une fois posé le décor, avec la mise en évidence des facteurs de contingence objectifs et structurels (petite taille, multiculturalisme) ou subjectifs et comportementaux (clientcentrisme, mimétisme par rapport au privé) qui s'exercent sur l'administration de Dubaï, il est temps de voir quel SPPP a été structuré dans le service public dubaïote.

\section{Le Système de Pilotage de la Performance Publique de Dubaï}

Tout système de pilotage de la performance comprend classiquement (Ferreira et Otley, 2009) : - la formulation des objectifs stratégiques et des indicateurs finaux de réussite;

- la mise en évidence des chaînes de causalité qui permettent de les atteindre et des critères de mesure du déploiement de la stratégie au niveau des activités;

- la détermination des niveaux de performance requis dans chaque centre de responsabilité pour atteindre les objectifs de l'entreprise;

- le système incitatif d'intéressement des managers et des employés couplé à la déclinaison des objectifs dans l'organisation;

- la détermination des flux d'information nécessaires pour mettre en œuvre la stratégie et pour l'adapter aux modifications de l'environnement.

Il s'agit maintenant d'analyser comment ces différents aspects sont mis en œuvre dans les administrations de Dubaï.

\subsection{Des objectifs stratégiques formalisés et des chaînes d'indicateurs liés}

Dubaï s'est adressée à la société de consulting Palladium, associée dès l'origine à l'élaboration du balanced scorecard par Norton et Kaplan. Un audit a été fait, des recommandations fournies et l'outil est maintenant décliné dans toute l'administration. L'appareil administratif de Dubaï s'appuie sur le plan stratégique, élabore des cartes cognitives et met en place une batterie d'indicateurs, avancés pour certains et ultimes pour les autres, mesurant la réalisation de la performance :

«Au KHDA, nous avons une carte stratégique qui est une étape en amont du BSC. La carte stratégique est la partie stratégique du BSC. Elle permet de définir les relations de cause à effet entre les éléments constituant la stratégie. Comment vous assurez-vous que les moyens mis en place vous donnent la stratégie que vous voulez? L'implémentation de la stratégie devient ainsi plus facile. Ensuite, nous avons des plans opérationnels pour chaque département. Ces plans sont issus de la stratégie de KHDA qui est elle-même inspirée du plan stratégique de Dubaï. » (KHDA-Dr K)

Ceci se retrouve dans toutes les directions et tous les services administratifs de Dubaï. Ainsi DNRD rend des comptes sur les objectifs stratégiques suivants : fournir le plus haut niveau de services ; maintenir la confiance des clients ; utiliser efficacement les ressources financières et technologiques; investir dans les ressources humaines et enfin encourager la 
créativité. Pour chaque objectif, il y a un indicateur et la direction de la stratégie consolide tous les indicateurs. Par exemple, Dubaï Airport veut devenir le leader mondial des aéroports. Pour cela, ses objectifs sont principalement : répondre à la croissance des compagnies aériennes comme Émirates Airlines et Fly Dubaï en assurant une bonne capacité d'accueil ; maintenir un niveau élevé de service à la clientèle (DA occupe la $28^{\mathrm{e}}$ position au niveau mondial pour ce critère); garantir la sureté et la sécurité des passagers et des avions ; retenir les personnes compétentes et les développer; améliorer les revenus.

L'administration de Dubaï fonctionne donc comme une administration de missions, s'adaptant aux orientations stratégiques du moment et déclinant en permanence les priorités de l'État dans son fonctionnement.

\subsection{Des budgets de programmes}

Une réforme mise en place en 2007 avec l'aide de cabinets conseils anglo-saxons de renommée mondiale a établi un mode de gestion budgétaire fondé sur les indicateurs de performance. L'objectif est d'articuler les objectifs politiques et leur traduction comptable :

«Le budget utilisé dans le gouvernement de Dubaï est un budget de programme, budget basé sur la performance et sur les résultats. Mais cela n'exclut pas l'utilisation du budget traditionnel (par lignes de dépenses, par comptes comptables). La préparation du budget est faite sur la base des programmes. L'exécution et le contrôle de budget se font par "line item» (par comptes comptables). Le budget est fait top-down (planification) et bottom-up (détermination du coût). Les deux à la fois pour une meilleure communication entre le top management et les managers opérationnels. Les idées viennent d'en haut et la construction se fait d'en bas. » (DOF - M. A)

La démarche employée est assez similaire sur ses principes à ce que la LOLF commence à mettre en place en France (Lambert et Migaud, 2006) :

«Nous appliquons le budget par objectifs qui est lié à nos objectifs stratégiques. Nos objectifs stratégiques sont traduits en objectifs départementaux. Chaque département fait son budget en fonction de ces objectifs. Chaque ligne budgétaire est liée à l'un des objectifs stratégiques que ce soit la capacité et la croissance, la sureté et sécurité, l'amélioration des revenus, l'amélioration du service client, etc. ». (DA - M.R)

Les révisions et ajustements budgétaires sont assez souples dans le SPPP :

«On n'est pas autorisé à dépasser le budget. Mais il y a une certaine flexibilité. On fait des transferts de budgets entre lignes budgétaires, entre rubriques et entre programmes. On demande l'autorisation de DOF en cas de transfert de budgets entre rubriques. En cas de besoin de fonds supplémentaires, on envoie une lettre officielle à DOF avec une justification des raisons et DOF nous accorde un budget supplémentaire. » (DP - Colonel S)

Ceci doit être mis en œuvre dans chaque administration, par exemple à l'éducation :

«Chez KHDA, nous avons le budget de programmes (program budgeting). C'est un budget qui lie les ressources aux résultats de chaque programme. Le budget est approuvé par projets. À l'intérieur du projet, il y a une structure de coûts. Ce qui est important, ce sont les projets et leurs objectifs. » (KHDA - M.P)

Pourtant certains cadres regrettent des manques de compétences en matière comptable dans les départements mais aussi au service financier. Il semble que la culture de la mesure ne soit pas encore vraiment développée et que les KPI ne reflètent qu'imparfaitement les 
activités. Un cadre dirigeant du département financier du gouvernement de Dubaï explique les faiblesses du dispositif :

"Je ne suis pas très satisfait car les administrations ne nous envoient que des rapports financiers. Il n'y a pas de rapports qui montrent la performance et qui font le lien entre le financier et les résultats. Par exemple, si on prend l'administration de la santé, ils nous envoient un rapport incluant les dépenses en salaires, en frais opérationnels, en immobilisations... mais ils n'envoient pas combien d'opérations du cour, combien d'accouchements, combien de visites, etc. On a besoin d'une autre dimension. On connaît les dépenses mais on a besoin de savoir ce que l'on fait avec ces dépenses pour être convaincu que ce qui a été dépensé est bon. » Et il explique : "L'Angleterre qui a une bonne structure financière et des agents financiers compétents a pris onze ans pour réaliser correctement une budgétisation axée sur la performance. » (DOF - Mr A)

\subsection{Benchmarking externe et émulation interne}

Des progrès importants, rendus nécessaires par les difficultés économiques subies par Dubaï en 2008, ont été réalisés dans la période récente :

"Avant la crise, il y avait une abondance de ressources. Il y avait une sorte d'indifférence. Il y avait une certaine extravagance. On faisait des projets dont on n'avait pas vraiment besoin. Après la crise, la culture de rationalisation des dépenses publiques a augmenté. Ils ont appris beaucoup de choses de la crise. Il y a des administrations qui ont beaucoup diminué leurs dépenses opérationnelles sans affecter leur qualité de service.»(DOF-M.A) En externe, le benchmarking (Bowerman et al., 2001) est développé par de nombreuses administrations comme l'administration de transport aérien :

«Nous envoyons des collègues à d'autres aéroports comme les aéroports de la Corée du Sud qui sont parmi les meilleurs aéroports au niveau mondial. Nos collègues préparent des rapports détaillés sur les domaines à améliorer. Nous faisons aussi des benchmarking avec des organisations n'opérant pas dans l'aviation. » (DA - M.R)

En interne, on développe aussi l'émulation entre les services :

«Le gouvernement de Dubaï veut toujours être le numéro 1. Il y a un plan stratégique pour Dubaï qui est distribué à toutes les administrations et autorités gouvernementales. Il y a le DGEP [Dubaï Government Excellence Program] qui crée une certaine compétition entre les administrations. Chaque administration essaye d'être excellente et de présenter ce qu'elle a de meilleur. Comme Cheikh Mohamed Ben Rachid a dit : dans la course vers l'excellence, il n'y a pas de limites ou de ligne finale. Il s'agit d'une amélioration continue. Il ne faut pas dire que ça y est, c'est fini, j'ai atteint mes objectifs et je suis le top des tops. Si maintenant tu es le premier, un jour un autre va venir te dépasser. » (DED-M.M)

\subsection{Certifications et audits}

DNRD est certifié ISO 9001. Il en est de même pour la plupart des administrations. C'est ainsi le cas du développement économique :

«Nous avons un certificat de qualité ISO. On le renouvelle tous les 3 ans. Les auditeurs viennent vérifier toutes les procédures et si toutes les conditions de qualité sont respectées. » (DED-M.M) 
À Dubaï Airports, il y a un département assurance qualité qui s'assure que les procédures sont respectées et qu'elles sont revues annuellement. Dans le cadre de la certification ISO, des auditeurs externes viennent également vérifier que les procédures sont respectées. Par ailleurs, dans des domaines critiques comme la sureté et la sécurité, des évaluateurs externes sont recrutés pour venir s'assurer que ce qui se passe en réalité respecte les procédures.

Le SPPP de Dubaï s'appuie sur les auditeurs internes. C'est ainsi vrai pour l'État-civil :

"Nous avons un audit interne qui audite tout ce qui est financier et aussi tout ce qui est procédures et processus. Il contrôle même les transactions dans les centres (le temps d'attente de chaque client et le temps que prend chaque agent pour faire une transaction). Il suggère des solutions et des applications pour améliorer les procédures. »(DNRD - M. H) Mais on recourt aussi systématiquement à des audits externes :

"Pour les audits externes, il y a l'audit du gouvernement de Dubaï, le FAD (Financial Audit Departement) et la cour des comptes fédérale. Ils auditent tout: les notes internes, les processus, tout ce qui est financier, etc.» (DNRD - M. H)

Les résultats sont malgré tout mitigés :

"Quelques processus sont formalisés mais pas tous. Il y a des procédures établies qu'il faut suivre à la lettre mais les gens font ce qu'ils veulent. C'est une grande bataille. On n'en finit jamais. C'est un travail continuel. » KHDA-M.P)

\subsection{Un ensemble d'incitations liées à la performance}

On évalue la performance individuelle des employés à travers un système que l'on appelle IPA (Individual Performance Agreement). Les employés sont évalués sur la base de l'atteinte des objectifs qui leur sont assignés au début de l'année et en se basant aussi sur leurs compétences. Réaliser une bonne évaluation n'est pas toujours facile :

«Nous appliquons le même cadre d'évaluation que les autres autorités. Nous avons des objectifs et nous avons des compétences. $70 \%$ pour les objectifs et $30 \%$ pour les compétences. Pour chaque employé, il y a des objectifs à réaliser avec des cibles et des mesures. Le seul souci dont on souffre, et souffrent aussi les autres administrations, c'est l'évaluation des compétences. Mesurer les compétences comportementales (comme traiter avec un client, savoir communiquer) est difficile. Mais nous faisons beaucoup d'efforts pour que le manager évalue son subordonné d'une bonne manière. » (DED - M. Sa)

Le système d'incitation est basé sur le mérite. L'évaluation de l'employé et sa notation influent sur l'augmentation de son salaire. Les notations vont de 1 à 4 (même notation dans tout le gouvernement de Dubaï) : 1 = Inférieur aux attentes ; 2 = Répond aux attentes ; 3 = Dépasse les attentes ; 4 = Dépasse largement les attentes. Il y a des pourcentages imposés par le gouvernement auxquels on ne peut déroger : $5 \%$ des employés doivent avoir la note $1 ; 75 \%$ des employés obtiennent la note $2 ; 15 \%$ des employés la note 3 et pas plus de $5 \%$ des employés la note 4 . Les employés qui ont des notes élevées peuvent présenter leur dossier de nomination au DGEP pour la catégorie de l'employé excellent. Il y a alors quatre critères à respecter : l'innovation, l'engagement, l'accomplissement et l'apprentissage continu. L'évaluation a des impacts directs et indirects sur la rémunération :

"Il y a un lien entre le système d'évaluation et le système d'incitation. Nous accordons des bonus pour les employés excellents. Nous accordons des certificats de reconnaissance. Nous donnons des prix financiers et non financiers. Nous donnons des congés 
comme récompense. Nous faisons des cérémonies pour rendre hommage aux employés performants. En intranet, nous publions une lettre de remerciement et d'appréciation de l'employé excellent. Nous faisons des promotions etc. »(DP - Colonel S)

Comme les syndicats permettant de faire remonter les insatisfactions ou de représenter le point de vue autonome des fonctionnaires n'existent pas à Dubaï, le climat social et la satisfaction au travail sont suivis avec attention :

«Onfait des enquêtes auprès de nos employés. On mesure leur satisfaction et leur engagement. Nous recevons les résultats et chaque manager en collaboration avec le département des ressources humaines doit trouver des solutions si le taux d'engagement de ses employés est faible. Par exemple, les employés travaillant dans le département financier s'ennuient car ils font des tâches répétitives. Pour remédier à cela, on a un plan de rotation. Ils passent 6 mois dans différentes sections (Budget, Reporting, Payements, etc.) pour apprendre de nouvelles choses. Nous essayons toujours d'améliorer l'engagement et la satisfaction de nos employés. » (DA - M.R)

Pour compléter le tableau donné par nos interlocuteurs, responsables de départements dans l'administration de Dubaï, il faut signaler que les salaires de base de la fonction publique sont soumis à une différenciation selon la nationalité, la loi prévoyant une retraite et un salaire plus élevé de $25 \%$ pour les Émiratis par rapport aux expatriés à niveau de poste et d'expérience égaux (mais eux touchent une indemnité à leur départ de l'organisation). Par ailleurs les expatriés n'ont le droit de résider à Dubaï que s'ils y ont une activité économique. S'ils perdent leur travail, ils perdent aussi leur droit de résidence à Dubaï. Il en ressort un faible sentiment d'appartenance et de sécurité et un fort taux de rotation des expatriés.

Ainsi les composantes de base du SPPP de Dubaï ont été présentées : objectifs stratégiques et indicateurs liés; budgets de programmes; mise en concurrence externe et interne, systématique et permanente; certifications et audits ; dispositif d'intéressement des services et des employés. Une analyse synthétique peut maintenant être effectuée de ce mode de gestion.

\section{Conclusion}

Ancienne colonie britannique, Dubaï a hérité des techniques de gestion publique anglosaxonnes et le cadre de gestion mis en place par l'équipe de direction autour du Cheikh relève totalement de l'approche du New Public Management. Dans la mise en œuvre, elle est confrontée à d'inévitables difficultés et limites comme montré précédemment. Des progrès sont encore nécessaires :

"Ce qui doit être amélioré, c'est la culture du résultat et de la responsabilité. Il y a des administrations qui font cela d'une bonne manière mais pas toutes les administrations. Nous avons encore des efforts à faire pour arriver à cela. » (DOF-M.A)

Même chose au niveau des individus :

"La culture de la mesure n'est pas appréciée par tous les employés. Parfois les mesures sont bénéfiques à certains employés, mais parfois elles les exposent. » (DA-M.R)

Par ailleurs plusieurs interlocuteurs laissent apparaître une insatisfaction en matière de prise de décision : beaucoup de «boards » cohabitent; les organigrammes sont souvent elliptiques; le gouvernement de Dubaï a un fonctionnement beaucoup moins centralisé que celui de Singapour par exemple ; l'informel et le délibératif sont omniprésents. Le statut des 
fonctionnaires, en majorité expatriés, apparaît moins valorisé qu'à Singapour souvent cité comme référence (Quah, 2010). On voit donc bien les failles et les améliorations possibles du système de pilotage administratif de Dubaï.

L'évaluation de l'impact des politiques publiques ne peut se juger que sur la durée et avec un horizon à moyen terme. Ceci amène un directeur à indiquer :

«En cinq ans, on a fait pas mal de choses. Maintenant est-ce qu'on est performant au niveau des " outcomes » sur le long terme? Je ne sais pas. ».

$\mathrm{Vu}$ la jeunesse de l'administration publique de Dubaï, les résultats globaux ne sont pas encore vraiment évaluables. Toutefois il semble que dans l'ensemble le service public fonctionne plutôt mieux que dans les autres pays comparables de la sous-région :

"Si vous nous comparez aux autres administrations de la région, nous sommes largement au-dessus. Si vous nous comparez aux pays les plus avancés, nous avons encore beaucoup à apprendre. » (KHDE-M.D)

Sur place les gens sont convaincus de la nécessité d'avoir un gouvernement aussi moderne et efficace que possible, car contrairement à la plupart des gouvernements de la sous-région, Dubaï ne peut pas s'appuyer sur la rente pétrolière. C'est un hub de services qui ne se développe que si la qualité de tous les services, publics comme privés, est là.

Bouckaert et Halligan (2008) ont proposé un cadre conceptuel pour comparer les différents modes de management de la performance publique dans les pays de l'OCDE. Ils prennent en compte les aspects liés à la collecte de l'information, à la transformation et à l'intégration de cette information dans les outils de gestion et à l'usage de l'information dans les processus de prise de décision. Ils en déduisent quatre stades de développement correspondant : 1/ à l'administration de la performance (vision bureaucratique); 2 / au management des performances (approche fonctionnelle) ; 3/ au management de la performance (vision globale et intégrée) et 4/ à la gouvernance de la performance (interactions avec les parties prenantes dans la société). Si on se réfère à cette catégorisation, Dubaï relève manifestement du modèle (3) : un mode de gestion managérial cohérent et complet mais avec une implication citoyenne réduite.

Cette recherche s'est focalisée sur la conception des systèmes de pilotage de la performance et sur la façon dont les équipes dirigeantes de l'administration de Dubaï abordent et évaluent la structuration et l'instrumentation de la gestion publique de la cité-État. Elle a permis de comprendre la cohérence de cette démarche, son relatif succès mais aussi son côté très contingent. Nous pensons avoir ainsi contribué à la compréhension fine et contextualisée de certaines caractéristiques des approches fondées sur le New Public Management. Ce cadre de référence théorique et pratique très porteur n'est aujourd'hui probablement pas encore assez pris en considération au sein de la communauté francophone de recherche en management public.

Pour le moment, d'autres aspects sont laissés de côté qui seront l'objet d'études ultérieures : l'approfondissement de la question de l'appropriation des outils de gestion par les managers et acteurs de terrain; une comparaison approfondie avec d'autres micro-États comme Luxembourg et Singapour; la réflexion sur la façon dont le système de pilotage de la performance de Dubaï a été impacté par la crise économique. 


\section{Bibliographie}

ABERBACH J.D., CHRISTENSEN T., (2005), Citizens and Consumers, an NPM Dilemma, Public Management Review 7 (2), 225-245.

ARMSTRONG H., de KERVENOAEL R., LI X., READ R., (1998). À Comparison of the Economic Performance of Different Microstates, and Between Microstates and Larger Countries, World Development 26 (4), 639 656.

BENEDICT B., (1967). Problems of smaller territories, Athlone Press, London.

BIYGAUTANE M., AL YAHYA K., (2010). Knowledge Management in Dubai's Public Sector : Opportunities and Challenges, Dubai School of Government.

BIYGAUTANE M., AL YAHYA K., (2011). The Current State of Knowledge Management in Dubai's Public Sector Organizations : Assessment and Recommendations, Dubai School of Government.

BOUCKAERT G., HALliGAN J., (2008). Managing Performance, International Comparisons, Routledge, London.

BOWERMAN M., BALL A., FRANCIS G., (2001). Benchmarking as a Tool for the Modernization of Local Government, Financial Accountability \& Management 17 (4), 321-329.

CROWARDS T., (2002). Defining the Category of 'Small'States, Journal of International Development 14 (2), 143-179.

CURMI L., (2009). Governance and Small States, Bank of Valletta Review 40.

DAVIDSON C.M., (2008). Dubai the Vulnerability of Success, Hurst publishers Ltd, London.

DEPARTMENT OF FINANCE, (2009 a). Program Budgeting, Government of Dubai.

DEPARTMENT OF FINANCE, (2009 b). Performance Based-Program Budgeting for Dubai Government, Government of Dubai.

DRIESKENS B., (2007). Global City, Tribal Citizenship : Dubai's Paradox, Cities of the South. Citizenship and Exclusion in the 21st Century, 261-292.

DUBAI STATISTICS CENTER, (2010). Dubai statistical yearbook 2010, Government of Dubai.

DUMORTIER B., LAVERGNE M., (2002). Doubaï : ville du pétrole ou projet métropolitain post-pétrolier, Annales de Géographie 623, 41-59.

ELASHMAWY S., (2007). Étude des finances publiques à la lumière de la stratégie de Dubaï (2007-2015), Department of Finance - Government of Dubaï (document en arabe).

EL KAHLOUT Z., (2010). Dubai Government Excellence Program : A Success Story, Middle East Quality Association 1 (3).

EL SHIMY N., (2008). From Free Zones to Special Economic Zones - The UAE Case Study, The Higher Corporation for Specialized Economic Zones.

FARRUGIA C., (1993). The Special Working Environment of Senior Administrators in Small States », World Development 21 (2), 221-226.

FERREIRA A., OTLEY D., (2009). The Design and Use of Performance Management Systems : an Extended Framework for Analysis, Management Accounting Research 20, 263-282.

GIBERT P., (1986). Management public, management de la puissance publique, Politiques et Management Public 4 (2), 89-123.

GLASSNER J., (2004). Du bon usage du concept de cité-État? Journal des africanistes 74 (1-2), 35-48.

GOVERNMENT OF DUBAI, (2011). Highlights Dubai Strategic Plan 2015.

HEARD-BEY F., (1999). Les Émirats arabes unis, Karthala, Paris.

HOFSTEDE G., (1983). National Cultures in Four Dimensions a Research-Based Theory of Cultural Differences among Nations, Studies of management and organizations 13 (1-2), 46-74.

HOOD C., (1991). A Public Management for All Seasons, Public Administration Review 69 (1), 3-19. 
IRIBANE P., (1989), La logique de l'honneur, Seuil, Paris.

KATZENSTEIN P., (2003). Small States and Small States Revisited, New Political Economy 8 (1).

LAMBERT A., MIGAUD D., (2006). La loi organique relative aux lois de finances (LOLF) : levier de la réforme de l'État, Revue française d'administration publique 117, 11-14.

LAVERGNE M., (2002). Dubaï ou la métropolisation incomplète d'un pôle en relais de l'économie monde, Cahiers de la Méditerranée.

LAVERGNE M., (2009). Dubaï, utile ou futile? Portrait d'une ville rêvée à l'heure de la crise, halshs-00375151, version 1 .

LAUFER R., BURLAUD A., (1980). Management public : gestion et légitimité, Dalloz Gestion, Paris.

LOOTAH R., OKAN G., (2006). Dubai Government Case Study, OECD / UNDP Meeting.

MAKHARITA R., (2005). Innovations in Public Administration: the Case of Dubai Government, UNDP Programme for Governance in the Region of Arab States, UNDP-POGAR.

MARCHAL R., (2001). Doubaï : cité globale, CNRS-Editions, Paris.

MEYSSONNIER F., (1997). Nature et outils du management public, in LE DUFF Le maire entrepreneur? Presses Universitaires de Pau.

MEYSSONNIER F., TAHAR C., (2011). Le contrôle des temps et des délais dans les services communaux, congrès de l'Association Francophone de Comptabilité (Montpellier).

OXFORD BUSINESS GROUP, (2008). The Report : Dubai 2008.

QUAH, (2010). Public Administration Singapore-Style, Emerald Group Publishing Limited, London.

RANDMA-LIIV T., (2002a). A Small Civil Service in Transition : The Case of Estonia, Public Administration and Development 21, 41-51.

RANDMA-LIIV T., (2002b). Small States and Bureaucracy : Challenges for Public Administration, Trames 6 (4), 374-389.

REGNIER P.T., (1987). Singapour et son environnement régional : étude d'une cite-État au sein du monde malais, Presses Universitaires de France, Paris.

SARAPU K., (2010), Comparative Analysis of State Administrations : The Size of State as an Independent Variable, Halduskultuur - Administrative Culture, 11 (1), 30-43.

SAXENA N.C. (2011). Virtuous cycles, the Singapore Public Service and National Development, Ministry of foreign Affairs.

SEVIC Z., (2001). Politico-Administrative Relationship in Small States, Bank of Valetta Review 23.

SIMONS R., (1995). Levers of Control, Harvard Business School Press, Harvard.

THORHALLSSON B., (2000). The Role of Small States in the European Union.

VINE P., (2010). Les Émirats Arabes Unies 2010 , Trident Press Limited, Cronulla.

ZAMPICCOLI L., (2011). Le management public : approches conceptuelles et enjeux de pilotage, Gestion et Management Public 9, mai, revue en ligne. 
Annexe :Nature et mode de réalisation des entretiens

\begin{tabular}{|c|c|c|c|}
\hline Services & $\begin{array}{l}\text { Personnes } \\
\text { rencontrées }\end{array}$ & Position & $\begin{array}{l}\text { Entrée dans } \\
\text { l'administration } \\
\text { dubaïote }\end{array}$ \\
\hline $\begin{array}{l}\text { Knowledge and Human } \\
\text { Development Authority } \\
\text { (KHDA) }\end{array}$ & $\begin{array}{l}\text { Docteur K. } \\
\text { Docteur D. } \\
\text { M. P. }\end{array}$ & $\begin{array}{l}\text { Directeur général } \\
\text { Directeur de la performance } \\
\text { Directeur financier }\end{array}$ & $\begin{array}{l}2006 \\
2007 \\
2007\end{array}$ \\
\hline $\begin{array}{l}\text { Dubaï Airport } \\
\text { (DA) }\end{array}$ & M. R. & Responsable financier & 2004 \\
\hline $\begin{array}{l}\text { Dubaï Economic } \\
\text { Development } \\
\text { (DED) }\end{array}$ & $\begin{array}{l}\text { M. M. } \\
\text { M. S h. } \\
\text { M. Sa. }\end{array}$ & $\begin{array}{l}\text { Directeur des services support } \\
\text { (Finance, RH, Achats) } \\
\text { Directeur financier } \\
\text { Responsable de l'évaluation de la } \\
\text { performance }\end{array}$ & $\begin{array}{l}1993 \\
1996 \\
2004\end{array}$ \\
\hline $\begin{array}{l}\text { Dubaï Police } \\
\text { (DP) }\end{array}$ & Colonel S. & Directeur financier & 1998 \\
\hline $\begin{array}{l}\text { Department of } \\
\text { Naturalisation and } \\
\text { Residency Dubaï } \\
\text { (DNRD) }\end{array}$ & M. H. & Directeur financier & 2007 \\
\hline $\begin{array}{l}\text { Department Of Finance } \\
\text { (DOF) }\end{array}$ & M. A. & $\begin{array}{l}\text { Ancien Directeur du budget } \\
\text { du gouvernement de Dubaï } \\
\text { Actuellement Directeur des études } \\
\text { financières à DOF }\end{array}$ & 1996 \\
\hline
\end{tabular}

L'étude a été menée par deux chercheurs : une chercheuse membre de l'administration de Dubaï et un chercheur extérieur. Un large accès à tous les documents administratifs internes ainsi qu'une connaissance interne fine des modes de fonctionnement et de la réalité de l'administration de Dubaï ont été possibles grâce au positionnement du premier chercheur. Et une distance critique par rapport au terrain a pu être préservée grâce au regard du second chercheur. 


\begin{tabular}{|c|c|c|c|c|}
\hline Formation & Nationalité & $\begin{array}{l}\text { Langue de } \\
\text { l'entretien }\end{array}$ & $\begin{array}{l}\text { Durée de } \\
\text { l'entretien }\end{array}$ & $\begin{array}{c}\text { Période } \\
\text { de l'entretien }\end{array}$ \\
\hline $\begin{array}{l}\text { Doctorat en } \\
\text { informatique } \\
\text { Doctorat en gestion } \\
\text { Master en gestion }\end{array}$ & $\begin{array}{l}\text { Emirati } \\
\text { Emirati } \\
\text { Italien }\end{array}$ & $\begin{array}{l}\text { Anglais } \\
\text { Anglais } \\
\text { Français }\end{array}$ & $\begin{array}{l}2 \text { heures } \\
2 \text { heures } \\
2 \text { heures }\end{array}$ & $\begin{array}{l}\text { Février } 2012 \\
\text { Février } 2012 \\
\text { Février } 2012\end{array}$ \\
\hline $\begin{array}{l}\text { Comptable Public } \\
\text { Agréé (CPA) }\end{array}$ & Égyptien & Anglais & 2 heures & Avril 2012 \\
\hline $\begin{array}{l}\text { Licence en } \\
\text { comptabilité (Master } \\
\text { en cours) } \\
\text { Licence en } \\
\text { comptabilité } \\
\text { (Master en cours) } \\
\text { MBA }\end{array}$ & $\begin{array}{l}\text { Emirati } \\
\text { Emirati } \\
\text { Jordanien }\end{array}$ & $\begin{array}{l}\text { Arabe } \\
\text { Arabe } \\
\text { Arabe }\end{array}$ & $\begin{array}{l}2 \text { heures } \\
45 \text { minutes } \\
45 \text { minutes }\end{array}$ & $\begin{array}{l}\text { Avril } 2012 \\
\text { Avril } 2012 \\
\text { Avril } 2012\end{array}$ \\
\hline Licence en droit & Emirati & Arabe & 2 heures & Mai 2012 \\
\hline non communiqué & Emirati & Arabe & $1 \mathrm{~h} 30 \mathrm{~min}$ & Mars 2012 \\
\hline $\begin{array}{l}\text { Licence en } \\
\text { comptabilité }\end{array}$ & Égyptien & Arabe & 2 heures & Mars 2012 \\
\hline
\end{tabular}


